\title{
Simultaneous load and structural monitoring of a carbon fiber rudder stock: Experimental results from a quasi-static tensile test
}

Journal of Intelligent Material Systems and Structures 2019, Vol. 30(2) 272-282 (C) The Author(s) 2018 Article reuse guidelines: sagepub.com/journals-permissions DOI: 10.1 I 77// 045389XI8806392 journals.sagepub.com/home/jim (S)AGE

\author{
Klaus Neuschwander', Jochen Moll' (D, Vittorio Memmolo ${ }^{2,3}$, \\ Matthias Schmidt ${ }^{2}$ and Marcel Bücker ${ }^{4}$
}

\begin{abstract}
Carbon-fiber-reinforced plastics are widely used in lightweight marine structures due to their high strength and superior fatigue behavior. In this article, we will present an innovative methodology for simultaneous load and structural monitoring of a carbon-fiber-reinforced plastic rudder stock as part of a big commercial vessel. Experimental results are presented here from a quasi-static tensile test in which the load monitoring is performed using embedded strain sensors. Structural monitoring is based on high-frequency electromechanical impedance spectroscopy combined with dedicated signal processing and surface-mounted piezoelectric transducers. We have achieved the following results: (I) the demonstration of a hybrid monitoring system including load and structural monitoring, (2) successful embedding of strain gauges during composite manufacturing of the carbon-fiber-reinforced plastic rudder stock, (3) development of instrumentation hardware for multichannel electromechanical impedance measurements, and (4) successful damage detection by means of electromechanical impedance spectroscopy in thick carbon-fiber-reinforced plastic rudder stock samples exploiting strain data.
\end{abstract}

\section{Keywords}

Rudder stock, marine structures, electromechanical impedance, load and structural monitoring

\section{Introduction}

In more and more applications within maritime structures, multimaterial mixes are chosen to build efficient structures with advantages in weight and performance. Due to their high strength and especially their superior fatigue behavior, carbon-fiber-reinforced plastics (CFRPs) can be an appropriate material for highly loaded parts. This article considers a rudder stock in CFRP for a big commercial vessel, as shown in Figure 1, where the rudder blade needs to be connected to the stock by press fit. This press fit causes high stresses in the radial direction of the stock, which is perpendicular to the fiber direction of a wet filament wounded CFRP tube. As a consequence, the CFRP part of the rudder stock must be connected to metallic parts, which are able to couple the rudder blade to the stock. This leads to a high stress concentration between metallic and CFRP parts, so that these areas are often most critical (Piggott, 1989; Wang et al., 2017). In CFRP components with very high wall thicknesses, the stress state is often more complex compared to thin-walled
CFRP parts and it is difficult to predict the fracture mechanics. This means that a continuous load and structural monitoring for such thick CFRP components is strongly needed.

Several examples can be found in the literature where a load monitoring with embedded sensors is combined with structural health monitoring (SHM) of the same structure (Bosse and Lechleiter, 2016; Ling and Mahadevan, 2012; Yuan et al., 2006). Previous works on ship structures have mainly focussed either on load monitoring or structural monitoring. The most

\footnotetext{
'Department of Physics, Goethe University of Frankfurt am Main, Frankfurt am Main, Germany

${ }^{2}$ Fraunhofer LBF, Darmstadt, Germany

${ }^{3}$ Department of Industrial Engineering, University of Naples Federico II, Naples, Italy

${ }^{4}$ CCOR-Schäfer MWN GmbH, Renningen, Germany
}

\section{Corresponding author:}

Jochen Moll, Department of Physics, Goethe University of Frankfurt am Main, Max-von-Laue Strße I, 60438 Frankfurt am Main, Germany.

Email: moll@physik.uni-frankfurt.de 


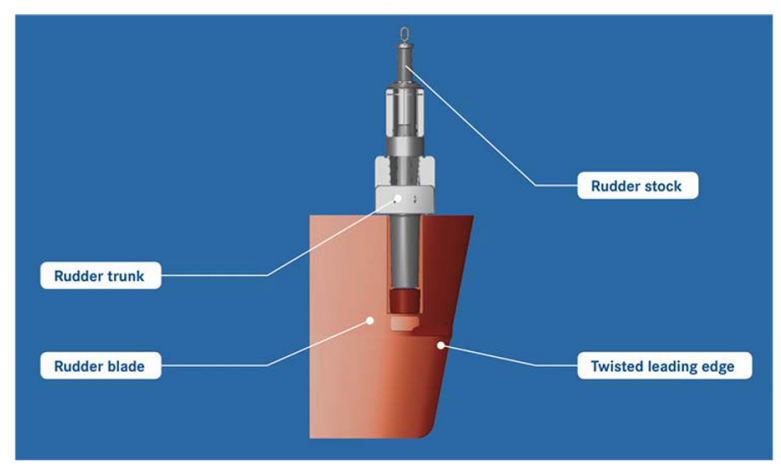

Figure I. Illustration of the rudder stock that transfers the torsional load from the steering mechanics to the blade (courtesy: Becker Marine Systems GmbH).

widely used principles for load monitoring are based on resistive or optical (fiber Bragg grating) strain gauges (Torkildsen et al., 2005). Moreover, accelerometers have been used by Phelps and Morris (2013) to detect global resonant behavior (whipping). Such loadmonitoring approaches enable the estimation of the structures' remaining life time and provide immediate feedback to the operator to allow real-time changes in maneuvering to minimize overstressing (Phelps and Morris, 2013).

However, several SHM techniques for ship structures have been reported in the literature. Okasha et al. (2010) show the integration of SHM in life-cycle performance assessment of ship structures under uncertainty. Model-based SHM of naval ship hulls is demonstrated in Stull et al. (2011). A wireless SHM system for submarine structures is presented in Nugroho et al. (2016). Damage identification in submerged shell structures by means of a differential evolution algorithm has been performed in Reed et al. (2013). A multipurpose monitoring system for icebreakers is presented in Zhirnov et al. (2016).

A promising SHM approach is given by the electromechanical impedance (EMI) method that was first described in Liang et al. (1994). The EMI method is based on the fact that the electrical impedance of a piezoelectric patch is linked to the mechanical impedance of the structure it is bonded to. In practice, the inverse of the complex impedance, the admittance $Y$, is often used. EMI has been successfully used for the detection of artificially induced delamination in composite materials (Ostachowicz et al., 2017). Structural damage in a grouted connection can also be detected by the EMI approach (Moll, 2018). A review on recent developments of the EMI method in different application domains is presented in Wandowski et al. (2017). To the best of the authors' knowledge, there is only one publication that employs the EMI method on ships by focussing on aluminum truss structures (Grisso, 2013).
It is well known that EMI spectra depend on external factors such as temperature (Wandowski et al., 2017), load of the structure (Annamdas et al., 2007; Taylor et al., 2013), and the thickness/stiffness of the adhesive (Islam and Huang, 2014; Tinoco and RosasBastidas, 2016; Tinoco and Serpa, 2011). The compensation of these effects is crucial to avoid false-positives in damage detection. Transducer failures by breakage or debonding as well as environmental factors have a higher impact on the imaginary part of the admittance than on the real part (Giurgiutiu and Zagrai, 2000).

The novel contributions of this article are given by the following items:

1. Demonstration of a hybrid monitoring system combining load and structural monitoring. Therefore, thick CFRP samples of a rudder stock have been manufactured and studied in a quasi-static tensile test.

2. Successful embedding of strain gauges during composite manufacturing of the CFRP rudder stock.

3. Development of instrumentation hardware for multichannel EMI measurements. In contrast to available devices, the presented measurement hardware supports high frequencies (up to $1 \mathrm{MHz}$ ) and high currents (up to $2 \mathrm{~A}$ ) so that highly attenuated materials such as the proposed CFRP rudder stock can be inspected.

4. Discussion of the successful combination of load and structural monitoring for damage detection by means of EMI spectroscopy in thick CFRP rudder stock samples. The dependency of the EMI signatures on the load cases can be eliminated by exploiting the load information from embedded strain gauges.

The remainder of this article is organized in the following way. Section "Measurement techniques for load and structural monitoring" presents the measurement techniques for load monitoring and structural monitoring. Subsequently, section "Experimental setup" describes the experimental setup of the quasi-static tensile test including a description of the rudder stock samples. Results for the hybrid monitoring concept are presented in section "Experimental results" followed by a discussion in section "Discussion." Finally, conclusions are drawn in section "Conclusion."

\section{Measurement techniques for load and structural monitoring}

\section{Load monitoring with embedded strain gauges}

The load monitoring is realized by embedding the strain gauge LI66-10/350 by HBM directly among composite layers. The sensor has a total size of $22 \mathrm{~mm}$ 

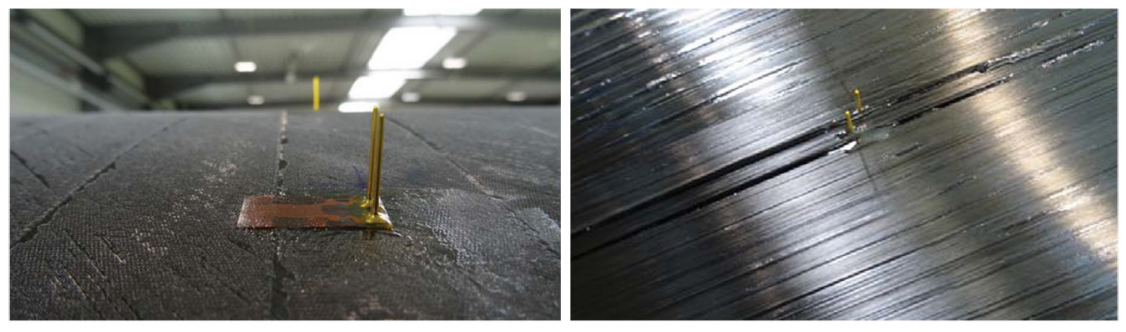

Figure 2. Positioning and embedding of the strain gauge in the middle plane of a CFRP tube.

Table I. Mechanical properties of the carbon epoxy lamina.

\begin{tabular}{lllllll}
\hline$E_{1}(\mathrm{GPa})$ & $E_{2}=E_{3}(\mathrm{GPa})$ & $G_{12}=G_{13}(\mathrm{GPa})$ & $G_{23}(\mathrm{GPa})$ & $\nu_{12}=\nu_{13}$ & $\nu_{23}$ & $\rho\left(\mathrm{kg} / \mathrm{m}^{3}\right)$ \\
\hline 175.0 & 9.8 & 4.3 & 3.2 & 0.25 & 0.43 & 1500 \\
\hline
\end{tabular}

$\times 10 \mathrm{~mm}$. For the sake of integration, a CFRP tube is manufactured in two different steps. In the former, the laminate is wound and cured up to the thickness where the sensors are then placed. On the emerging surface, the sensors are located in the central part of the final specimen (see Figure 7). An epoxy glue (Hardman Double Bubble Epoxy), chemically very similar to the resin, was used to fix the strain gauges for the wet filament winding of the CFRP. The thickness of the adhesive film is few microns. The sensors have connection pins facing outwards (see Figure 8), making it possible to wind the second part of the CFRP laminate without losing access to the sensor. After that, the sensor is positioned on the laminate plane, where the interesting strain occurs, and the laminate of the second part is wound and cured up to the final thickness. Figure 2 shows the glued sensors on the left and the laminate before final curing step on the right.

The sensor layout allows the embedding during manufacturing as well as an efficient structural behavior and a reasonable metrology response. The continuous load monitoring can be achieved by an embedded sensor when it does not impair the host structure, and the strain information is correctly correlated to the actual load. Several promising results have been reported while investigating fatigue life, interlaminar shear (ILS) resistance, and interlaminar fracture toughness energy $\left(G_{I C}\right)$ on test specimens designed with similar materials and manufacturing technique (Horoschenkoff et al., 2006). Unfortunately, there is no investigation available in the literature on the second mode of failure and flexural stiffness, which are crucial to design bendingloaded structures such as the rudder stock. For this reason, the bending response is preliminary analyzed here focusing on the structural performance and the sensor metrology response.

To achieve a comprehensive characterization of the integrated strain gauges, structural and metrology investigations have been carried out using several standard specimens $(250 \mathrm{~mm} \times 40 \mathrm{~mm} \times 2 \mathrm{~mm})$ made of carbon-epoxy material. High-tensile carbon fibers and epoxy resin adopted for manufacturing the rudder stock are used to obtain unidirectional laminae whose properties are reported in Table 1. Eight plies are symmetrically stacked in a quasi-isotropic plate to obtain 20 specimens which prevent any mechanical coupling between in-plane load and out-of-plane deformations, and vice versa (Jones, 1998).

Among them, 10 specimens are equipped to perform the end-notched flexure (ENF) tests oriented to evaluate the second mode toughness $G_{I I C}$ (Sham Prasad et al., 2011). They are obtained introducing a $13-\mu \mathrm{m}$ polytetrafluoroethylene (PTFE) film along the neutral plane according to the relevant standard (ASTM D7905/D7905M-14, 2014). Forces are applied to the specimen through a dedicated three-point bending fixture under displacement-controlled loading provided by a 10-KN Zwick-Roell ${ }^{\circledR}$ Z010 testing machine. A record of the applied force versus center roller displacement is obtained using the real-time analog-to-digital (A/D) X-Y recorder of the machine and setting a sampling rate of $50 \mathrm{~Hz}$. The $G_{I I C}$ is obtained using the compliance calibration (CC) method (Jagannathan et al., 2015), which is believed to represent the critical strain energy release rate for crack growth from the insertion film (Bru et al., 2016). The average $G_{I I C}$ results from five replicate tests performed on flat and integrated structures each. They are found to be about 217 and $201 \mathrm{~J} / \mathrm{m}^{2}$ respectively, with a scatter of about $7 \%$ which excludes any critical effect on the fracture behavior while embedding sensors for the specimen under test.

Using the same setup, 10 further tests are carried out according to the standard D7264/D7264M-15 (2015). The flexural modulus of elasticity is computed as the chord modulus of elasticity from load and displacement records. The average value from five replicate tests on 


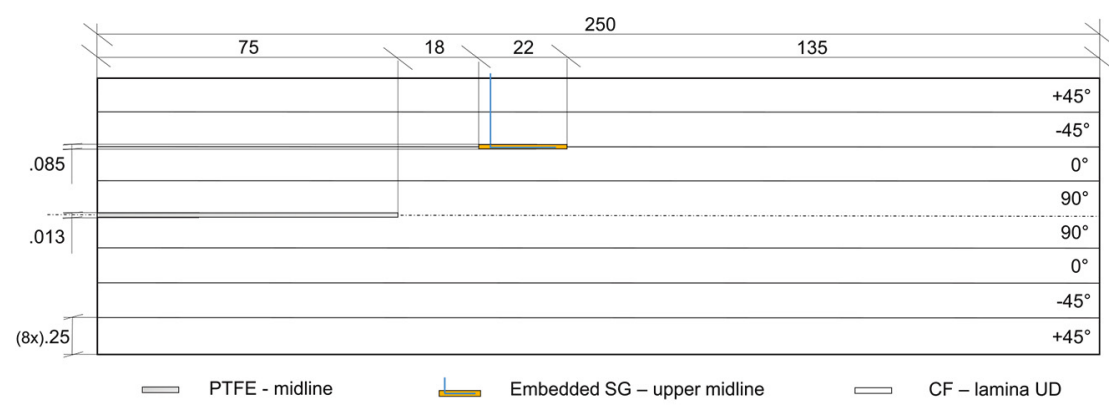

Figure 3. Illustration of the stacking sequence $\left(+45^{\circ} /-45^{\circ} / 0^{\circ} / 90^{\circ}\right)_{S}$ of the test specimen showing the location of the strain gauge and the pins. The thickness of the structure is $\approx 2 \mathrm{~mm}$ (dimensions in $\mathrm{mm}$ ).

flat and integrated structures each are found to be about 12.5 and $13.5 \mathrm{GPa}$, respectively. Despite the scatter of about $9 \%$ is still acceptable, it unexpectedly suggests that the flexural stiffness increases while inserting the sensor due to the vertical configuration of the pins (see Figure 3 for the location of the strain gauge and the pins).

The crucial point for the object of this research is the metrology efficiency of the embedded sensor. A measurement comparison is carried out performing flexural tests on fine integrated specimens according to the standard D7264/D7264M-15 (2015). The outcomes from embedded strain gauges are opportunely scaled to obtain the maximum deformation expected on the upper surface, where another conventional strain gauge is mounted. The surface-mounted strain gauge LY46$10 / 350 \mathrm{~A}$ by HBM is chosen to match the characteristics of the embedded solution. The PICAS ${ }^{\circledR}$ multichannel compact amplifier system is used to amplify the strain gauges with a quarter bridge configuration, while the oscilloscope OnoSokki ${ }^{\circledR}$ CF3600 digitizes the voltage signals with a sampling rate of $10 \mathrm{~Hz}$. A typical result obtained is reported in Figure 4. The butterfly distribution of the strain through the thickness of the specimen leads to different voltage outputs. Hence, an equivalence factor $\beta$ is computed considering that distribution in order to obtain the equivalent deformation depicted. Different measurements are carried out on five specimens, showing a repeatability as well as reproducibility of the experiments. Table 2 compares $\beta$ with the ratio $(\alpha)$ between the maximum deformations measured by both strain gauges. In detail, when $\alpha$ is greater than $\beta$, the conventional strain gauge returns a deformation greater than the equivalent data coming from the embedded sensor. The equivalence factor of the strain gauge depends on the thickness of the specimen $(h)$ and the distance from the surface $(d)$ which is here computed accounting also the thickness of the strain gauge $(t)$. The percentage error reported in the same table demonstrates the very slight difference obtained while using the latter one. It is worth noting that the error due to a possible misalignment of the sensors is here

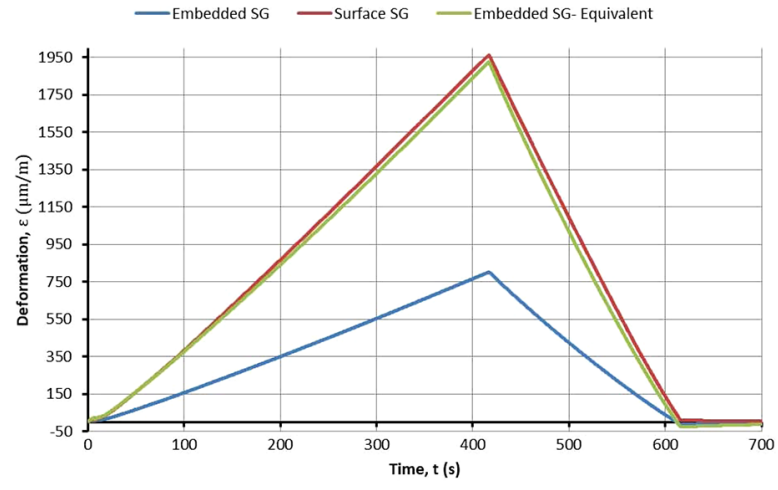

Figure 4. Strain histories measured with embedded (blue) and surface-mounted (red) strain gauge. The equivalent value (green) takes into account the thickness of the sensor $(t=0.085 \mathrm{~mm})$.

Table 2. Maximum deformation measured with strain gauges under displacement-controlled loading, equivalence factor $(\beta)$, and ratio between maximum deformations $(\alpha)$.

\begin{tabular}{llllll}
\hline Specimen & $\varepsilon_{\text {surf }}(\mu \varepsilon)$ & $\varepsilon_{\text {int }}(\mu \varepsilon)$ & $\beta$ & $\alpha$ & $E(\%)$ \\
\hline$R_{1}$ & 2323 & 977 & 2.4 & 2.38 & 0.83 \\
$R_{2}$ & 2192 & 894 & 2.4 & 2.45 & 2.083 \\
$R_{3}$ & 2083 & 883 & 2.4 & 2.36 & 1.67 \\
$R_{4}$ & 2085 & 885 & 2.4 & 2.32 & 3.33 \\
$R_{5}$ & 1962 & 802 & 2.4 & 2.45 & 2.083 \\
\hline
\end{tabular}

strongly limited by the lamination sequence used (Horoschenkoff et al., 2006).

\section{EMI spectroscopy}

A measurement device was designed explicitly for ultrasound SHM in high-attenuation materials. It is capable of performing both EMI and pitch-catch measurements on up to 12 channels by time-division multiplexing. High excitation voltages up to $100 \mathrm{~V}_{\mathrm{pp}}$ and currents up to $2 \mathrm{~A}$ are provided by a PD200 piezo driver manufactured by PiezoDrive. The $\mathrm{A} / \mathrm{D}$ conversion is done using 


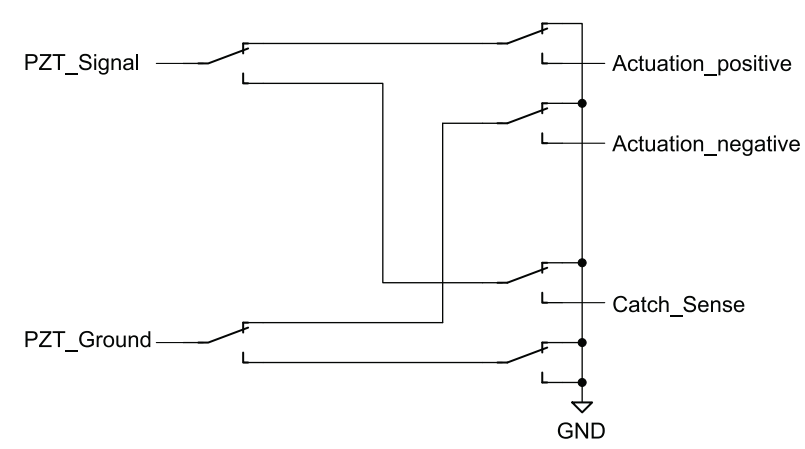

Figure 5. Schematic diagram of one multiplexer channel.

a HS3 Handyscope at 10 MSPS. The device is specified for EMI actuation up to $1 \mathrm{MHz}$. Each multiplexer channel consists of three double-pole-double-throw mechanical relays to switch both actuation and ground signals (Figure 5). This complex design is only necessary for the added pitch-catch functionality, which requires the ability to route two independent signals to arbitrary piezo channels. All unused signal traces are grounded to avoid crosstalk between the channels. The piezos are connected with coaxial cables, since the high-powered signals would be strong enough to influence other equipment without proper shielding. The EMI circuit uses a shunt resistor to measure the current through the transducer while simultaneously measuring the actuation voltage. The basic design idea follows the work from Peairs et al. (2004), with some major modifications to handle the high-amplitude signals. A very low shunt resistor value of $0.05 \Omega$ is used to minimize power dissipation, which requires a more sophisticated amplification circuit (Figure 6). The excitation voltage is scaled down by a factor of 10 to avoid crosstalk between the voltage and current channels inside the analog-to-digital converter (ADC) unit. The voltages at the ADC unit are thus given by

$$
V^{C h 1}(t)=\frac{R_{f 1}}{R_{g 1}} V_{\text {piezo }}(t) \quad V^{C h 2}(t)=\frac{R_{f 2}}{R_{g 2}} R_{\text {shunt }} I_{\text {piezo }}(t)
$$

For each frequency, a single-tone sinusoidal waveform is generated and the two input channels are sampled for $15 \mathrm{~ms}$. The values of $V_{P Z T}$ and $I_{P Z T}$ are then transferred to the frequency domain using the Goertzel algorithm, because only one frequency is of interest (the signal generator frequency), which in general will not correspond to an exact FFT-bin. This allows to both choose the correct frequency and save computational power compared to an FFT by only calculating one component. The admittance for this excitation frequency is then defined as

$$
Y\left(f_{i}\right)=\frac{I_{P Z T}\left(f_{i}\right)}{V_{P Z T}\left(f_{i}\right)}
$$

The above computation is performed in real time on the device. The resulting admittance values are streamed through a network interface.

During EMI measurements, temperatures are measured using DS18B20 sensors, which provide a relative resolution of $0.0625^{\circ}$. An intermediate version of this device was presented in Neuschwander et al. (2017).

\section{Experimental setup}

A drawing and a picture of the rudder stock specimen is shown in Figures 7 and 8, respectively. The sample consists of a CFRP core with rectangular cross-section and a metal part on both ends which is screwed to correctly fix the specimen on a Schenck ${ }^{\circledR} 2500-\mathrm{kN}$ electrohydraulic testing machine. Moreover, a metallic pin is connected to the steel flanges. This metal pin is part of the actual rudder stock design to connect its CFRP parts to the metallic component that couples the rudder blade

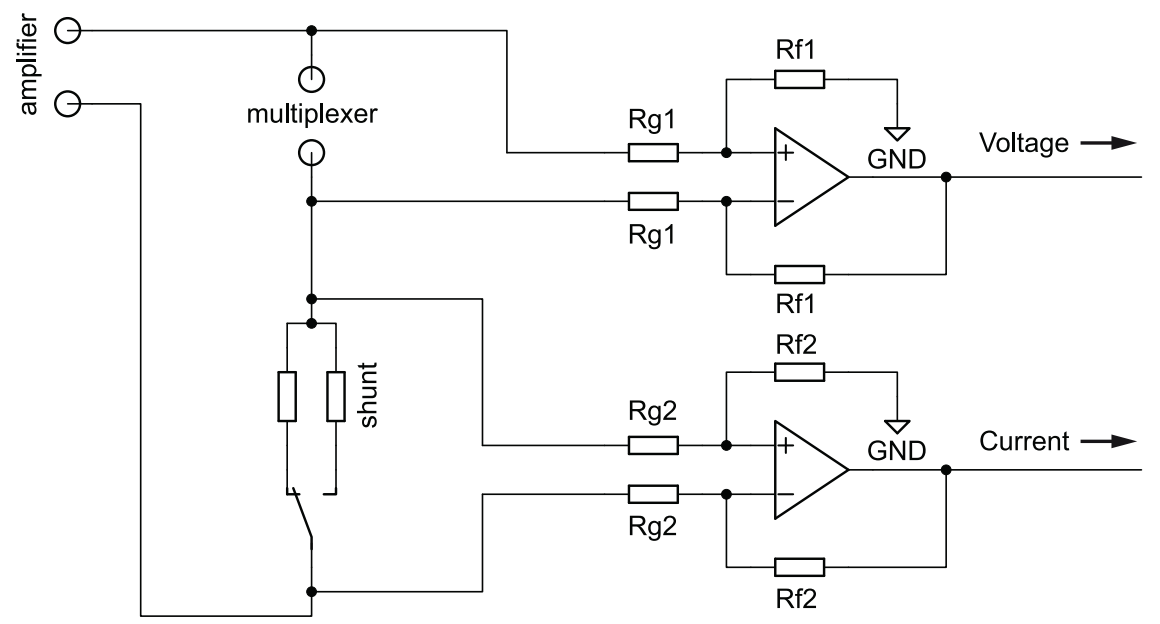

Figure 6. Schematic diagram of EMI circuit. 

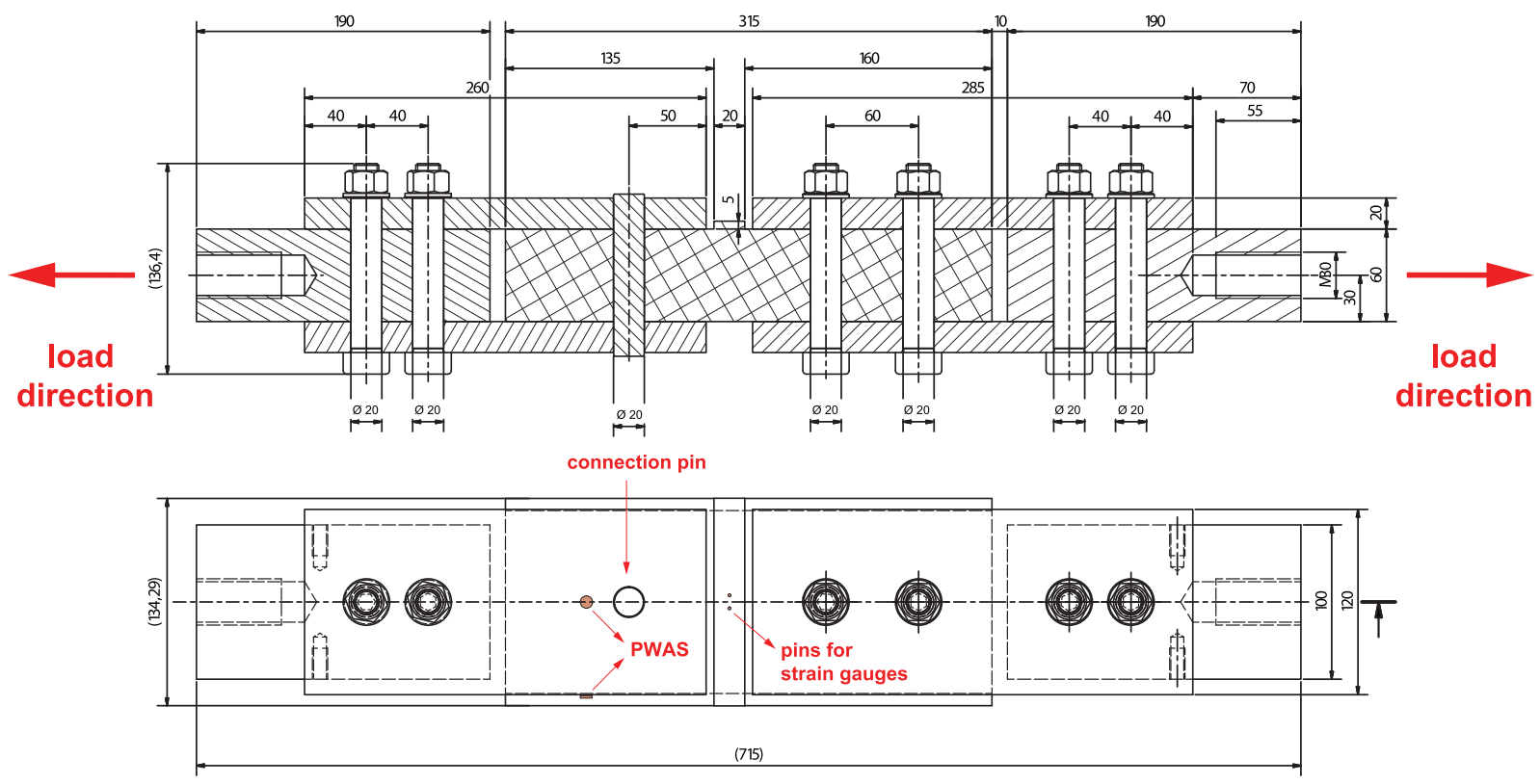

Figure 7. $C A D$ drawing of the test specimen including the sensor locations. Since the drawing is rotated by $90^{\circ}$ with respect to the experimental setup, the load direction is in horizontal direction.

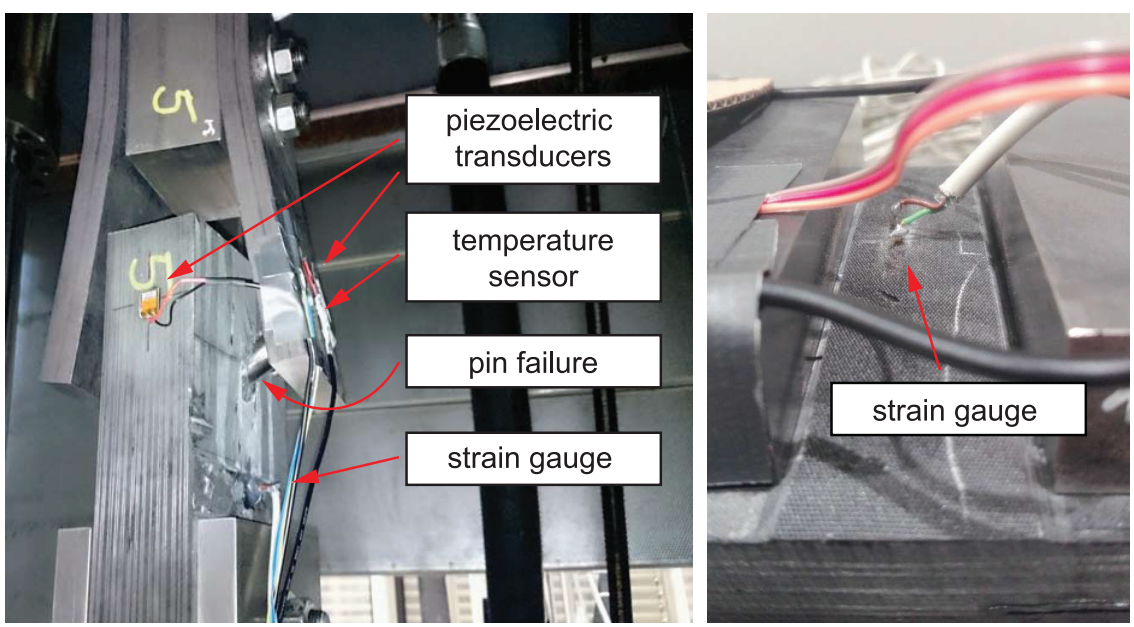

Figure 8. Left: photo of the rudder stock specimen after the tensile test instrumented with two piezoelectric transducers, an embedded strain gauge and a temperature sensor; right: close-up view of the embedded strain gauge.

to the stock. The detection of pin failure is important for safe operation of the ship.

Two piezoelectric transducers are mounted to the surface of the specimen. A rectangular DuraAct transducer (PI Ceramic $\mathrm{GmbH}$ ) with a size of $16 \mathrm{~mm} \times$ $13 \mathrm{~mm} \times 0.5 \mathrm{~mm}$ is glued on the CFRP material using cyanoacrylate glue with a thickness of a few microns. A circular PIC255 transducer (PI Ceramic $\mathrm{GmbH}$ ) with a diameter of $10 \mathrm{~mm}$ and a thickness of $0.25 \mathrm{~mm}$ is placed on the steel part together with a DS18B20 temperature sensor that records the current temperature on the specimen's surface so that temperature-related effects can be identified.

Figure 9 shows a photo of the data acquisition system for strain and EMI. The strain gauge is connected to an amplifier which sends the amplified DC strain signal to an ADC. In this case, we have used a Handyscope HS3 from TiePie Engineering which supports remote control from MATLAB. The spectra of both EMI channels and the current temperature are forwarded to the computer over Ethernet. All measurement data are synchronized and stored in a single HDF5-file. 


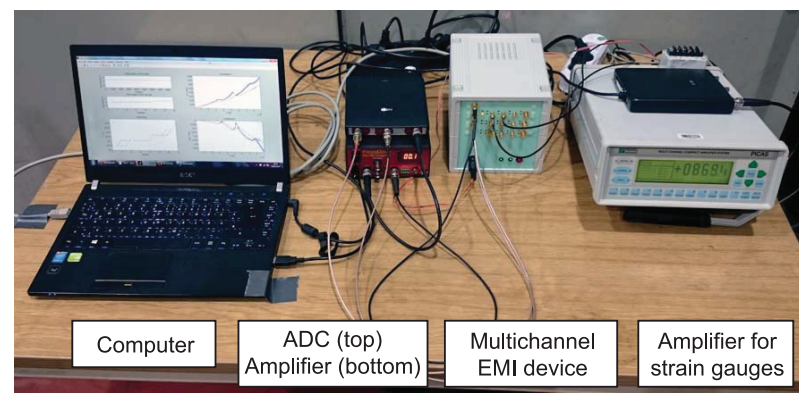

Figure 9. Photograph of the data acquisition equipment during the quasi-static tensile test.

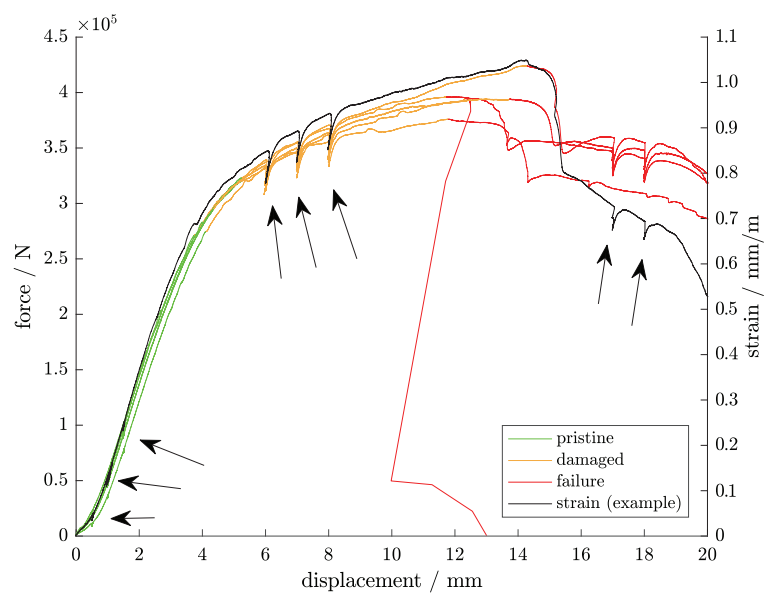

Figure 10. Force-displacement graph of testing the rudder stock specimens divided in intact (green), damaged (orange), and failure state (red). One strain example from the embedded strain gauge is provided.

\section{Experimental results}

Figure 10 shows the force-displacement graphs of the tests for five rudder stock specimens that are used in this study. At the beginning, all curves show a nearly linear-elastic behavior, marked in green. At this stage, the specimen can be considered to be intact and no damage has occurred so far verified by visual inspection and the absence of acoustic emission events. With further increase in the load, a degradation of the stiffness, marked in orange, can be observed given by a change in the gradient of the force-displacement graphs (Puck, 1996; Schürmann, 2007). In the CFRP, a degradation of stiffness is caused by inter-fiber failure, that is, small cracks in the epoxy matrix. In the steel parts, stiffness degradation is caused by a plasticization of the material. Both phenomena are connected with non-reversible damage in the material, whose detection is the main goal of the study. When this stage occurs, a typical sudden drop in the curve slightly appears
(ASTM D7905/D7905M-14, 2014), but a further increase in the force is possible. It starts increasing again very quickly but the slope decreases very soon toward the total failure. When first severe cracks in the steel or first ruptures of carbon fibers occur, a drop in the force level can be detected. In this state, marked in red, the connection usually fails and is no longer able to hold force. In the real scenario, when the load is not driven by the displacement of a machine, a separation of the connected parts is possible and needs to be avoided in any case by detecting damage before the total failure level is reached. During the test, the procedures were paused for $180 \mathrm{~s}$ at the points marked with arrows to perform the EMI measurements without interference from the noises of the test machine. During these stops, a small relaxation of the force level can be observed, but the original level is reached very quickly after continuing the testing.

The strain curve depicted in Figure 10 shows the strain versus displacement trend from the sensor embedded in the specimen 4 . This example clearly demonstrates that the load can be monitored by means of embedded strain gauge until failure, providing correct information about increasing load, stops during loading procedure, and fatal failure. As discussed above, both the force and the strain versus displacement plots clearly show a sort of first-ply failure through a slight sudden drop in the curve (ASTM D7905/D7905M-14, 2014). This behavior is quite clear in Figure 10 from the strain distibution (around $0.7 \mathrm{~mm} / \mathrm{m}$ ). Further increasing the load, the deformation starts increasing again and very quickly toward the total failure but the slope of the curve decreases very soon due to an overall stiffness reduction.

All EMI spectra for specimen 4 during the whole experimental cycle are shown in Figure 11. The admittance values differ even without structural changes. This effect is consistently stronger for sensor 1 than for sensor 2 in all specimen, which indicates that the effect is not due to different transducers, but rather to the location on the structure. Both temperature and strain are possible sources for effects of this type. In the case of strain, the effect would be more prominent in steel due to the larger stiffness of CFRP. Both variables cannot be varied independently, so data from multiple specimen have to be combined to find consistent relationships to the spectrum changes. Only specimens 1 and 4 are used for the following analysis, because those are the only ones with reliable strain information and a full experimental cycle. The dependency can be quantified by comparing each curve to a reference curve, in this case, the first EMI measurement. Three independent variables $(\Re(Y), \Im(Y)$, and $f)$ are recorded for frequencies up to $900 \mathrm{kHz}$, and each of them is 

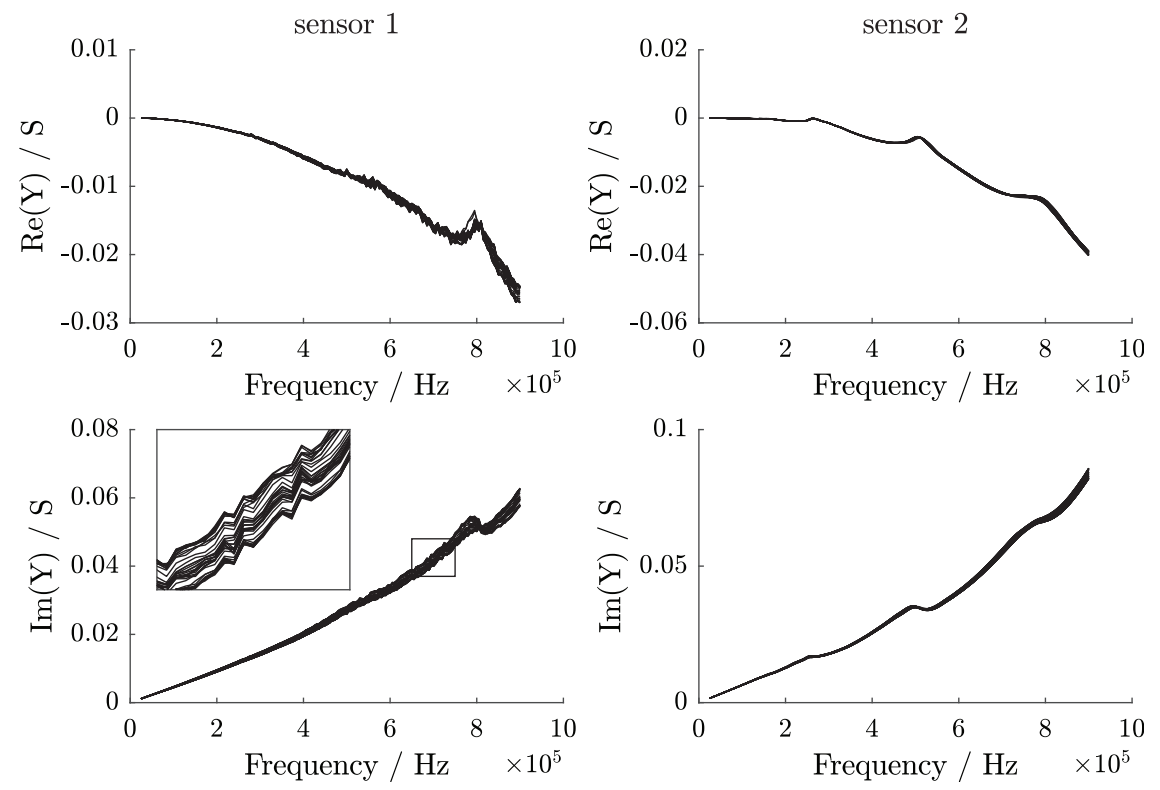

Figure I I. EMI spectra of specimen 4. During the experiment, the admittance values increase, resulting in multiple curves which only differ by a small scaling factor. Sensor I (on steel) shows one resonance and a finer structure with low amplitude, whereas sensor 2 (on CFRP) shows three broad resonances with high amplitude. At high frequencies, a negative conductance is displayed due to a phase shift inside the measurement circuit. This absolute phase shift does not have an influence on the relative changes of the spectrum that are of interest for damage detection.
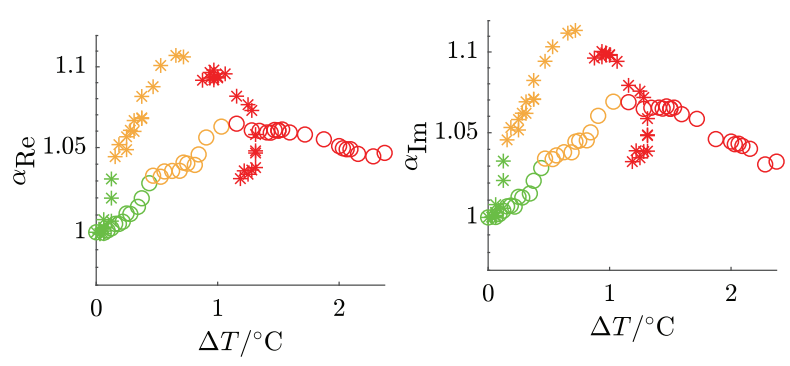

Figure 12. Scaling parameters for the first piezoelectric transducer on specimens I $\left(^{\circ}\right)$ and $4\left(^{*}\right)$ as a function of temperature change. Given by the small temperature variations during the experiment, the scaling parameters do not show a temperature dependency.

transformed by an affine transformation which has two parameters per variable, resulting in six independent parameters

$$
\begin{gathered}
\Re\left(Y^{\prime}\right)=\alpha_{\Re} \cdot \Re(Y)+\beta_{\Re} \\
\Im\left(Y^{\prime}\right)=\alpha_{\Im} \cdot \Im(Y)+\beta_{\Im} \\
f^{\prime}=\alpha_{f} \cdot f+\beta_{f}
\end{gathered}
$$

These parameters are found by fitting each curve onto the reference using the least-squares algorithm. This procedure is independent of the strain and temperature data. The parameters $\alpha_{\Re}$ and $\alpha_{\Im}$ are the ones relevant for the effect described above, because they scale in the vertical direction of the admittance graph.
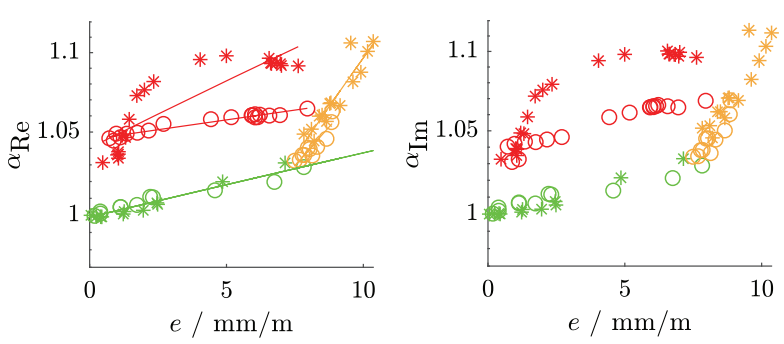

Figure 13. Scaling parameters for the first piezoelectric transducer on specimens $\mathrm{I}\left({ }^{\circ}\right)$ and $4\left(^{*}\right)$ as a function of strain e. Linear fits have been computed for the real part of the scaling parameters.

Plotting these parameters against the temperature yields two completely different curves, whereas the relationship to the strain is the same for both specimen before failure (cf. Figures 12 and 13). This leads to the conclusion that the scaling effect is due to strain, and not temperature. Therefore, local strain information has to be accessible to provide meaningful results for EMI measurements in thick CFRP samples under strong loading.

A linear regression model $\hat{\alpha}_{\Re}(e)$ has been derived for the intact specimens 1 and 4 (see green line in Figure 13). This model serves as a baseline for the definition of a damage indicator (DI) which is defined as the absolute difference between the scaling parameter $\alpha_{\Re}$ and the linear model. Mathematically, this relationship can be formulated for the $i$ th measurement as 


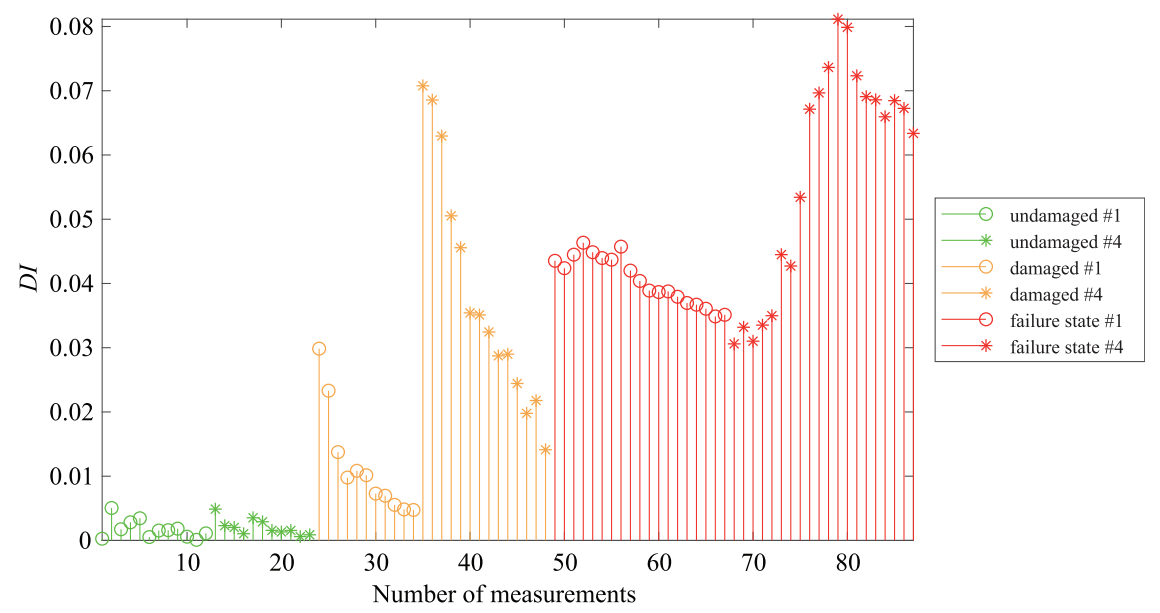

Figure I4. Damage indicator describing the healthy, damaged, and failure states of two test specimens, that is, specimens I (*) and $4\left({ }^{\circ}\right)$.

$$
D I(i)=\left|\hat{\alpha}_{\Re}(e)-\alpha_{\Re}(i)\right|
$$

In a similar way, the above procedure can also be applied for the imaginary part of the scaling parameter $\alpha_{\Im}$.

Figure 14 provides a quantitative analysis for the three structural states, that is, intact, damaged, and failure state based on the DI. It can be observed for both specimens 1 and 4 that the DI has a relatively small value for the undamaged state. The DI increases for the case of the damaged state proportionally to the applied load (marked in orange) and further increases for the failure state (marked in red). This quantitative analysis demonstrates that the proposed DI is able to detect structural damage in the presence of structural loading.

\section{Discussion}

The preliminary mechanical tests demonstrate the suitable integration process, the excellent metrological response of strain gauges when embedded among composite layers, and the low impact on mechanical properties. It is worth noting that the revealed small discrepancies are expected to decrease while increasing the thickness and number of plies, which is the case of the rudder stock. In addition, the lower deformation that involves the embedded sensor and the protection provided by the hosting structure increase its durability, making this technological solution feasible as well as suited for load monitoring during the lifetime of the composite rudder stock. Due to the high stresses involved in the composite part of the real-scale structure, a strain level beyond 4000 microstrains is indeed expected during operation. It can be reduced twice or more ( $\beta$ factor) while adopting the embedded strain gauge opportunely. This fact enhances the durability performances of the sensor, whose maximum strain is limited to around 1500-2000 microstrains. Of course, the strain expected at failure depends strongly on the combination of loads and on the position of the strain gauge with respect to the midline $(d / h)$. It is worth to achieve a maximum strain at the strain gauge lower than the limit using a $\beta$ factor close to 2 . This ensures that the sensor works correctly after failure and, generally, during lifetime so that the DIs can be assessed by further varying the strain level. Generally, by accommodating this parameter, it is possible to design and optimize a safer, reliable, and long-life load-monitoring system. The same discussion can be made for the piezoelectric sensors adopted for SHM, where the strain, depending on the combination of load, will be close to that aging at the external ply.

About the damage scenario simulated during experiments, due to the high loads engaged, the tensile test has been carried out in safe condition with a strong cabin around load machine. This prevented the on-line and detailed failure assessment. However, the acoustic emission during fiber failure and crack has been easily assessed by the operators and correlated to the applied displacement. Furthermore, the fibers pull out was visually evaluated and the total failure was assessed after test by visually inspecting the specimens. On top of that, the first failure (associated with the internal fiber crack) can be assessed from the typical sudden drop in both the force and the strain versus displacement curves (even visible in Figure 10). This trend, typical of composite laminate structure at first-ply failure, clearly defines the limit between healthy and first damaged condition and it is an efficient way to evaluate the SHM capabilities of the hybrid system.

It was demonstrated by the proposed tensile test that the EMI method is able to distinguish between the intact, damaged, and failure state of the rudder stock structure. It should be noted here that this study was conducted in a laboratory environment with controlled environmental conditions. When the methodology is 
applied in the industrial setting, a variety of changing ambient conditions will occur that are related, for example, to significant temperature variations, water loading, and mechanical vibrations. Further research is needed to study those additional effects and their influence on the proposed DIs.

A structural monitoring system also needs to define statistical thresholds and confidence intervals that characterize the intact structural state and provides information when the structure is damaged. This can be implemented in a training phase directly after the installation when the structure can be considered as intact.

\section{Conclusion}

In this article, we successfully demonstrated in a quasistatic tensile test that a combination of load monitoring and structural monitoring can be used to detect damage in thick composite rudder stock samples using a combination of embedded strain gauges and the EMI method. Therefore, strain gauges have been successfully embedded in the composite material. In addition, a multichannel EMI instrument was proposed that supports high frequencies (up to $1 \mathrm{MHz}$ ) and high currents (up to $2 \mathrm{~A}$ ) so that highly attenuated materials can be inspected. A DI was defined that is able to take the load information from the embedded strain gauges into account. By this means, the undamaged, damaged, and failure states of the structure can be distinguished. The results presented here show the potential for realizing this hybrid monitoring model for operational ship structures.

\section{Authors' note}

For more information about Jochen Moll, please visit http:// www.jochenmoll.de

\section{Declaration of conflicting interests}

The author(s) declared no potential conflicts of interest with respect to the research, authorship, and/or publication of this article.

\section{Funding}

The author(s) disclosed receipt of the following financial support for the research, authorship, and/or publication of this article: This research was supported by the Federal Ministry for Economic Affairs and Energy (grant nos 03SX422A and 03SX422B).

\section{ORCID iD}

Jochen Moll iD https://orcid.org/0000-0003-2299-2250

\section{References}

Annamdas VGM, Yang Y and Soh CK (2007) Influence of loading on the electromechanical admittance of piezoceramic transducers. Smart Materials and Structures 16(5): 1888-1897. Available at: http://stacks.iop.org/0964-1726/ $16 / \mathrm{i}=5 / \mathrm{a}=045$ ? key $=$ crossref.df20627aa4e380d84e6e $1 \mathrm{fc} 3 \mathrm{~b}$ $70 \mathrm{f} 5074$

ASTM D7905/D7905M-14 (2014) Standard test method for determination of the mode II interlaminar fracture toughness of unidirectional fiber-reinforced polymer matrix composites.

Bosse S and Lechleiter A (2016) A hybrid approach for structural monitoring with self-organizing multi-agent systems and inverse numerical methods in material-embedded sensor networks. Mechatronics 34: 12-37. Available at: http: //linkinghub.elsevier.com/retrieve/pii/S0957415815001464

Bru T, Hellstr m P, Gutkin R, et al. (2016) Characterisation of the mechanical and fracture properties of a uni-weave carbon fibre/epoxy non-crimp fabric composite. Data in Brief 6: 680-695.

D7264/D7264M-15 (2015) Standard test method for flexural properties of polymer matrix composite materials.

Giurgiutiu V and Zagrai A (2000) Damage detection in simulated aging-aircraft panels using the electro-mechanical impedance technique. In: Adaptive structures and material systems symposium, Orlando, FL, 5-10 November, pp. 349-358. New York: ASME.

Grisso BL (2013) Development of a structural health monitoring prototype for ship structures. Technical Report 468. Washington, DC: Ship Structure Committee.

Horoschenkoff A, Klein S and Haase K (2006) Structural integration of strain gauges. Technical Report. Darmstadt: HBM GmbH.

Islam MM and Huang H (2014) Understanding the effects of adhesive layer on the electromechanical impedance (EMI) of bonded piezoelectric wafer transducer. Smart Materials and Structures 23(12): 125037. Available at: http://stacks. iop.org $/ 0964-1726 / 23 / \mathrm{i}=12 / \mathrm{a}=125037$ ? $\mathrm{key}=$ crossref. 68 42bad636c8f7340ddff98bcefb3fle

Jagannathan N, Anil Chandra A and Manjunatha C (2015) Onset-of-growth behavior of mode II delamination in a carbon fiber composite under spectrum fatigue loads. Composite Structures 132: 477-483.

Jones RM (1998) Mechanics of Composite Materials (Materials Science and Engineering Series). New York: Taylor \& Francis.

Liang C, Sun F and Rogers C (1994) Coupled electromechanical analysis of adaptive material systemsdetermination of the actuator power consumption and system energy transfer. Journal of Intelligent Material Systems and Structures 8(4): 335-343.

Ling Y and Mahadevan S (2012) Integration of structural health monitoring and fatigue damage prognosis. Mechanical Systems and Signal Processing 28: 89-104. Available at: http://linkinghub.elsevier.com/retrieve/pii/S088832701 1003980

Moll J (2018) Damage detection in grouted connections using electromechanical impedance spectroscopy. Proceedings of 
the Institution of Mechanical Engineers, Part C: Journal of Mechanical Engineering Science. Epub ahead of print 30 January. DOI: 10.1177/0954406218764226.

Neuschwander K, Shrestha A, Moll J, et al. (2017) Multichannel device for integrated pitch catch and EMI measurements in guided wave structural health monitoring applications. In: 11th international workshop on structural health monitoring, Stanford, CA, 12-14 September, pp. 1723-1730. Lancaster, PA: DEStech Publications, Inc.

Nugroho WH, Purnomo NJH and Soedarto T (2016) An experimental work on wireless structural health monitoring system applying on a submarine model scale. Journal of Physics: Conference Series 776: 012094. Available at: http://stacks.iop.org/1742-6596/776/i=1/a = 012094?key = crossref.c5eada03f7b57a606bd3a3b0f13cd7c6

Okasha NM, Frangopol DM and Decò A (2010) Integration of structural health monitoring in life-cycle performance assessment of ship structures under uncertainty. Marine Structures 23(3): 303-321. Available at: http: //linkinghub.elsevier.com/retrieve/pii/S0951833910000389

Ostachowicz W, Malinowski P and Wandowski T (2017) Highlights and challenges in damage assessment of composite structures. In: 8th ECCOMAS thematic conference on smart structures and materials, Madrid, 5-7 June, pp. $1-15$.

Peairs DM, Park G and Inman DJ (2004) Improving accessibility of the impedance-based structural health monitoring method. Journal of Intelligent Material Systems and Structures 15(2): 129-139.

Phelps B and Morris B (2013) Review of hull structural monitoring systems for navy ships. Technical Report DSTOTR-2818. Australian Government Department of Defence. Available at: http://dspace.dsto.defence.gov.au/dspace/ handle/dsto/10246

Piggott M (1989) The interface in carbon fibre composites. Carbon 27(5): 657-662. Available at: http://linkinghub. elsevier.com/retrieve/pii/0008622389901991

Puck A (1996) Festigkeitsanalyse Von Faser-matrix-laminaten: Modelle Für Die Praxis. München: Hanser.

Reed H, Nichols J and Earls C (2013) A modified differential evolution algorithm for damage identification in submerged shell structures. Mechanical Systems and Signal Processing 39(1-2): 396-408. Available at: http://linking hub.elsevier.com/retrieve/pii/S0888327013000976

Schürmann H (2007) Konstruieren Mit Faser-kunststoffverbunden: Mit 39 Tabellen. VDI-[Buch], 2., bearb. und erw. aufl. Berlin: Springer.

Sham Prasad MS, Venkatesha CS and Jayaraju T (2011) Experimental methods of determining fracture toughness of fiber reinforced polymer composites under various loading conditions. Journal of Minerals and Materials Characterization and Engineering 10: 1263-1275.
Stull CJ, Earls CJ and Koutsourelakis PS (2011) Model-based structural health monitoring of naval ship hulls. Computer Methods in Applied Mechanics and Engineering 200(9-12): 1137-1149. Available at: http://linkinghub.elsevier.com/ retrieve/pii/S0045782510003427

Taylor SG, Park G, Farinholt KM, et al. (2013) Diagnostics for piezoelectric transducers under cyclic loads deployed for structural health monitoring applications. Smart Materials and Structures 22(2): 025024. Available at: http: //stacks.iop.org/0964-1726/22/i=2/a =025024

Tinoco HA and Serpa AL (2011) Bonding influence in the electromechanical (EM) admittance of piezoelectric sensors bonded to structures based on EMI technique. In: Proceedings of the 14th international symposium on dynamic problems of mechanics (DINAME XIV), Sao Sebastiao, Brazil, 13-18 March, pp. 335-344.

Tinoco HA and Rosas-Bastidas DA (2016) Experimental study of the debonding effects on the electromechanical impedance of piezo wafer active sensors. In: Proceedings of III international conference on advanced mechatronics, design and manufacturing technology, Cali, Colombia, April 2016, pp. 1-10.

Torkildsen HE, Grovlen A, Skaugen A, et al. (2005) Development and applications of full-scale ship hull health monitoring systems for the Royal Norwegian Navy. In: Recent developments in non-intrusive measurement technology for military application on model- and full-scale vehicles; Meeting proceedings RTO-MP-AVT-124, Neuilly-sur-Seine Cedex, France: North Atlantic Treaty Organisation, Research \& Technology Organisation, pp. 22-21.

Wandowski T, Malinowski PH and Ostachowicz WM (2017) Temperature and damage influence on electromechanical impedance method used for carbon fibre-reinforced polymer panels. Journal of Intelligent Material Systems and Structures 28(6): 782-798.

Wang ZY, Wang QY, Li L, et al. (2017) Fatigue behaviour of CFRP strengthened open-hole steel plates. Thin-Walled Structures 115: 176-187. Available at: http://linkinghub. elsevier.com/retrieve/pii/S0263823116306693

Yuan S, Lai X, Zhao X, et al. (2006) Distributed structural health monitoring system based on smart wireless sensor and multi-agent technology. Smart Materials and Structures 15(1): 1-8. Available at: http://stacks.iop.org/0964$1726 / 15 / \mathrm{i}=1 / \mathrm{a}=029$ ?key $=$ crossref.fbeda99af830284bb3ee $1 \mathrm{cbd} 67 \mathrm{a} 52 \mathrm{~d} 62$

Zhirnov A, Anufriev M, Pozhar N, et al. (2016) Multipurpose monitoring system for icebreakers: development, implementation, and testing. MATEC Web of Conferences 75: 04005. Available at: http://www.matec-conferences.org/10. 1051/matecconf/20167504005 